\title{
КОМПЬЮТЕРНОЕ МОДЕЛИРОВАНИЕ ПРОЦЕССА РАВНОКАНАЛЬНОГО УГЛОВОГО ПРЕССОВАНИЯ В МАТРИЦЕ НОВОЙ КОНСТРУКЦИИ
}

Одним из наиболее известных способов интенсивной пластической деформации (ИПД), позволяющим получать объемные заготовки с ультрамелкозернистой (УМЗ) структурой является равноканальное угловое прессование (РКУП). Несмотря на то, что этот метод ИПД известен уже более 40 лет [1], он до сих пор активно исследуется многими учеными [2-5]. С момента первого упоминания о РКУП до настоящего времени учеными из разных стран мира разработан целый ряд новых технологических схем реализации ИПД и инструментов для их осуществления [6-9], в основе многих из которых лежит именно принцип РКУП, однако подавляющее большинство этих идей не нашло широкого применения в промышленности. Причиной этому являются сравнительно небольшие размеры получаемых заготовок, дискретность процесса деформации и необходимость осуществления большого числа циклов деформирования для формирования УМЗ-структуры, т. е. все те недостатки, которые относятся именно к РКУП.

Для преодоления первых двух недостатков, уже не первое десятилетие ведется разработка так называемых «совмещенных» процессов [10-13], в которых действует принцип непрерывной деформации длинномерных образцов. Суть же последнего недостатка заключается в том, что для получения УМЗ-структуры в деформируемом материале необходимо обеспечить реализацию высоких степеней деформации для измельчения зерна, что достаточно сложно при небольшом количестве циклов деформирования. Увеличение же циклов деформирования ведет, как к увеличению энергозатрат, так и трудозатрат. Одним из вариантов решения данной проблемы на наш взгляд является совершенствование инструмента для равноканального углового прессования. При этом при разработке инструмента для РКУП новой конструкции нужно не забывать, что эффективным способом повышения проработки металла при РКУП является кантовка деформируемого образца. Так, в работе [14] всесторонне исследован данный фактор и доказано, что наиболее оптимальным является маршрут ВС, который предполагает кантовка на $90^{\circ}$ вдоль продольной оси образца после каждого цикла, т. е. смена горизонтального направления деформации в поперечном сечении на вертикальное.

Целью данной работы является исследование процесса деформирования в равноканальной ступенчатой матрице новой конструкции, отличительной особенностью которой является расположение промежуточного канала под двумя углами к входному и выходному каналам, т. е. деформация происходит не в горизонтальном или вертикальном направлениях, а в обоих одновременно (диагональное направление), в частности, исследование влияния величины угла наклона в поперечном направлении $\beta$ (рис. 1,6 ) на напряженно-деформированное состояние заготовки и энергосиловые параметры данного процесса. По сути, деформирование в матрице такой конструкции представляет собой обычное РКУ-прессование с частичной кантовкой образца. Схематично диагональное движение металла в данной матрице показано на рис. 1, а.

Для проведения МКЭ-моделирования равноканального ступенчатого прессования с использованием матриц с течением в 3 плоскостях в программе «КОМПАС» были построены 4 модели матриц. Первая матрица имела «классическую» равноканальную ступенчатую конструкцию, в которой направление течения металла менялось лишь в 2 плоскостях. Остальные 3 матрицы имели схожую с первой конструкцию, с той лишь разницей, что в них направление течения металла осуществляется не в двух, а в трех плоскостях. Угол стыка каналов в продольном направлении (угол $\alpha$ ) во всех матрицах принимался равный 135 градусов, как наиболее оптимальный при равноканальном ступенчатом прессовании [15]. Угол стыка каналов в поперечном направлении (угол $\beta$ ) в первой матрице был равен нулю, в остальных трех матрицах он был равен соответственно 15, 25 и 35 градусов (рис. 1, б). 


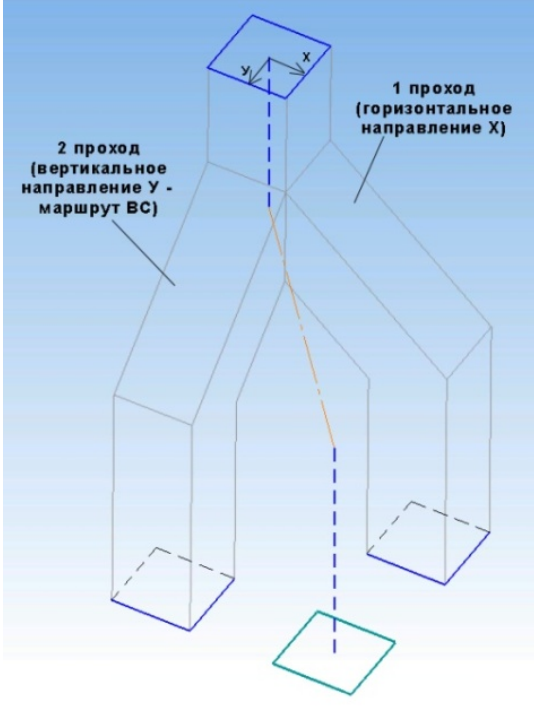

a

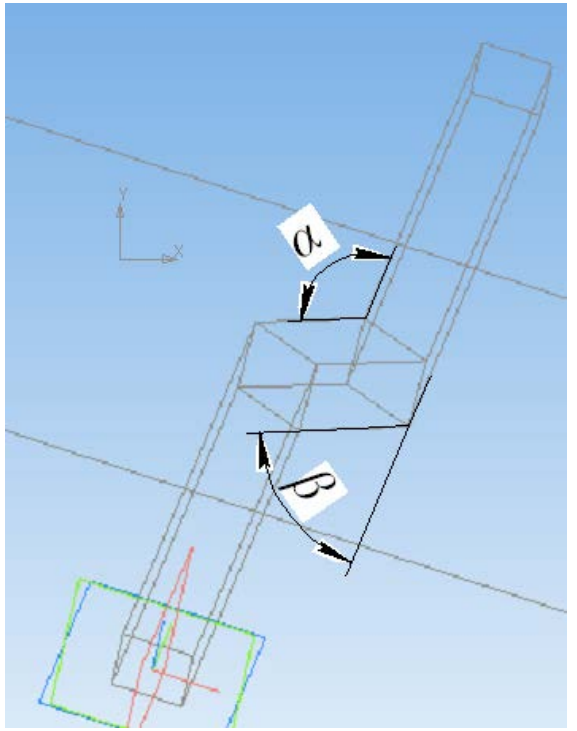

б

Рис. 1. Схема конструкции каналов в матрице с течением в 3 плоскостях

Исходная заготовка имела размеры 20 х 20 х 100 мм. На данную модель была нанесена сетка конечных элементов, равномерно распределенных по всему объему заготовки. Средний размер конечного элемента составил 1,3 мм, количество узлов - 30699, количество элементов - 96024. В качестве материала заготовки была выбрана сталь 35, нагретая до температуры $1100{ }^{\circ} \mathrm{C}$. Реологические свойства материала были взяты из базы данных Deform. Был выбран неизотермический тип расчета, т. е. помимо отдачи тепла инструменту, заготовка еще отдавала тепло в окружающую среду, температура которой была принята $20{ }^{\circ} \mathrm{C}$. При ранее проведенном исследовании равноканального ступенчатого прессования было установлено, что от значения коэффициента трения в матрице будет зависеть требуемое усилие, необходимое для стабильного протекания процесса деформирования. Для минимизации его значения было взято значение коэффициента трения равное 0,1 .

Для изучения напряженно-деформированного состояния (НДС) были рассмотрены следующие параметры:

1) Эквивалентная деформация Strain Effective (общая интенсивность деформаций). В общем случае при 3-х мерном течении металла все компоненты тензора деформации не равны нулю и должны быть рассчитаны. Однако, поскольку тензор деформации является объектом, который очень трудно визуально представить (визуализировать), то для практических целей был использован простой показатель интенсивности деформации, или так называемая эквивалентная деформация, которая включает в себя все компоненты тензора деформации в следующем виде:

$$
\varepsilon=\frac{\sqrt{2}}{3} \sqrt{\left(\varepsilon_{x}-\varepsilon_{y}\right)^{2}+\left(\varepsilon_{y}-\varepsilon_{z}\right)^{2}+\left(\varepsilon_{z}-\varepsilon_{x}\right)^{2}+\frac{3}{2}\left(\gamma_{x y}^{2}+\gamma_{y z}^{2}+\gamma_{z x}^{2}\right)},
$$

2) Главные напряжения Stress Max Principal $\left(\sigma_{1}\right)$, Stress Min Principal $\left(\sigma_{3}\right)$. Их значения находят по следующим формулам:

$$
\begin{aligned}
& \sigma_{1}=\frac{\sigma_{X}+\sigma_{Y}}{2}+\sqrt{\left(\frac{\sigma_{X}-\sigma_{Y}}{2}\right)^{2}+\tau_{X Y}{ }^{2}}, \\
& \sigma_{3}=\frac{\sigma_{X}+\sigma_{Y}}{2}-\sqrt{\left(\frac{\sigma_{X}-\sigma_{Y}}{2}\right)^{2}+\tau_{X Y}{ }^{2}} .
\end{aligned}
$$


Компонент $\sigma_{2}$ обычно не рассматривается, поскольку он является средним арифметическим от $\sigma_{1}$ и $\sigma_{3}$.

3) Эквивалентное напряжение Stress Effective (интенсивность напряжений), вычисляемое по формуле:

$$
\sigma=\frac{1}{\sqrt{2}} \sqrt{\left(\sigma_{x}-\sigma_{y}\right)^{2}+\left(\sigma_{y}-\sigma_{z}\right)^{2}+\left(\sigma_{z}-\sigma_{x}\right)^{2}+6\left(\tau_{x y}^{2}+\tau_{y z}^{2}+\tau_{z x}^{2}\right)},
$$

При этом рассматривались два этапа деформирования:

- первый этап - передний конец заготовки находится во втором канале матрицы;

- второй этап - передний конец заготовки находится в третьем канале матрицы.

\section{Результаты и обсуждение. Изучение деформированного состояния}

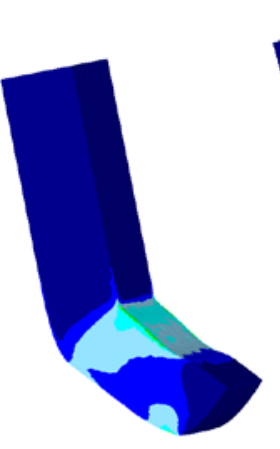

a)

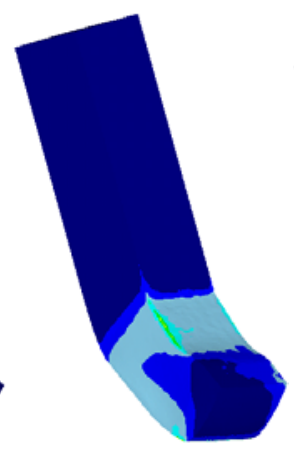

б)

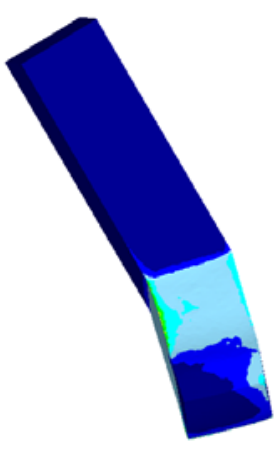

в)

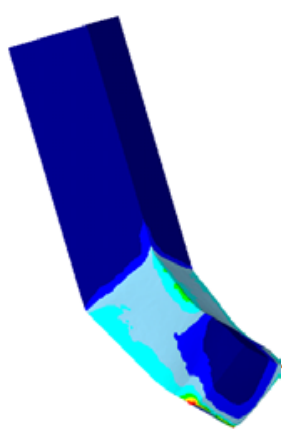

Strain - Effective ( $\mathrm{mm} / \mathrm{mm})$

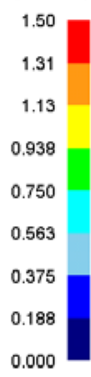

г)

$\mathrm{a}-$ матрица с углом $\beta=0^{\circ} ; \sigma$ - матрица с углом $\beta=15^{\circ}$;

в - матрица с углом $\beta=25^{\circ} ; \Gamma$ - матрица с углом $\beta=35^{\circ}$

Рис. 2. Распределение эквивалентной деформации на первом этапе деформирования

На первом этапе деформирования накопление эквивалентной деформации происходит при движении заготовки во втором канале матрицы. В матрице с углом $\beta=0^{\circ}$ распределение эквивалентной деформации имеет неравномерный характер (рис. 2, а), значение которой колеблется в диапазоне $0,2 \div 0,4$. С повышением значения угла $\beta$ (рис. 2 , б-г) распределение эквивалентной деформации по длине заготовки выравнивается, а его численное значение увеличивается. В матрице с углом $\beta=15^{\circ}$ значение эквивалентной деформации равно $0,45 \div 0,5$; в матрице с углом $\beta=25^{\circ}$ оно равно $0,55 \div 0,62$; в матрице с углом $\beta=35^{\circ}$ оно равно $0,7 \div 0,82$.

На втором этапе деформирования накопление эквивалентной деформации происходит при движении заготовки во втором и в третьем каналах матрицы. В матрице с углом $\beta=0^{\circ}$ распределение эквивалентной деформации, как и на первом этапе, имеет неравномерный характер (рис. 3, а), отчетливо видны зоны разных значений деформации, значение которой колеблется в диапазоне $0,7 \div 1$. С повышением значения угла $\beta$ (рис. 3 , б-г) распределение эквивалентной деформации по длине заготовки становится более равномерным, а его численное значение увеличивается. В матрице с углом $\beta=15^{\circ}$ значение эквивалентной деформации на втором этапе равно $0,75 \div 1,07$; в матрице с углом $\beta=25^{\circ}$ оно равно $0,85 \div 1,1$; в матрице с углом $\beta=35^{\circ}$ оно равно $0,92 \div 1,22$.

Главные напряжения являются одной из важнейших характеристик напряженнодеформированного состояния, поскольку при их рассмотрении появляется возможность изучения распределения растягивающих и сжимающих напряжений по всему объему деформируемой заготовки.

Главное напряжение $\sigma_{1}$ является преимущественно растягивающим, поэтому при его рассмотрении на заготовке отчетливо видны зоны напряжений с положительными значениями. 
На первом этапе деформирования в матрице с углом $\beta=0^{\circ}$ (рис. 4 , а) значение главного напряжения $\sigma_{1}$ находится в диапазоне $180 \div 220$ МПа. С повышением значения угла $\beta$ (рис. 4, б-г) значение главного напряжения $\sigma_{1}$ увеличивается. В матрице с углом $\beta=15^{\circ}$ значение $\sigma_{1}$ равно $240 \div 280$ МПа; в матрице с углом $\beta=25^{\circ}$ оно равно $280 \div 320$ МПа; в матрице с углом $\beta=35^{\circ}$ оно равно $300 \div 350$ МПа.

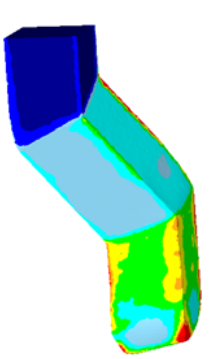

a

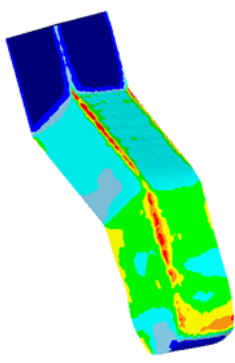

6

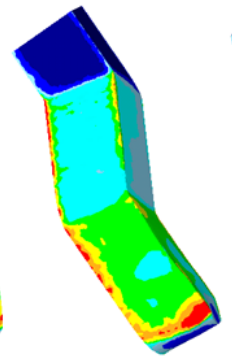

B

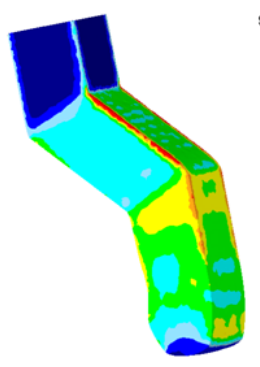

$\Gamma$

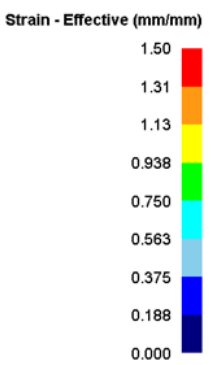

0.000

a-матрица с углом $\beta=0^{\circ} ; \sigma-$ с углом $\beta=15^{\circ} ; \mathrm{B}-$ с углом $\beta=25^{\circ} ; \Gamma-$ с углом $\beta=35^{\circ}$

Рис. 3. Распределение эквивалентной деформации на втором этапе деформирования

Изучение напряженного состояния

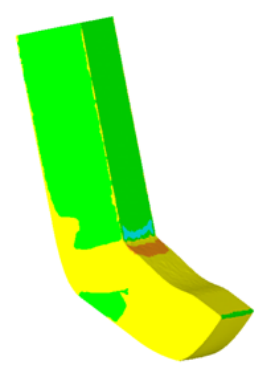

a

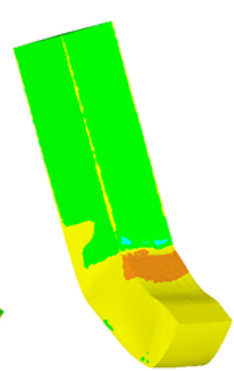

6

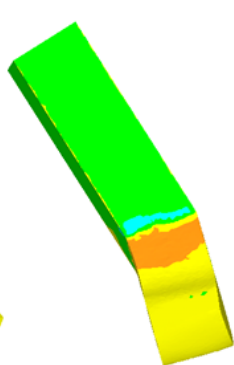

B

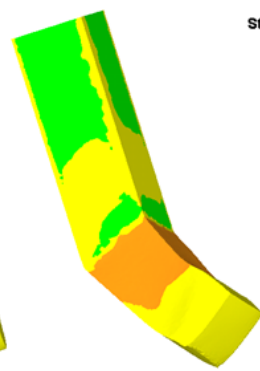

$\Gamma$

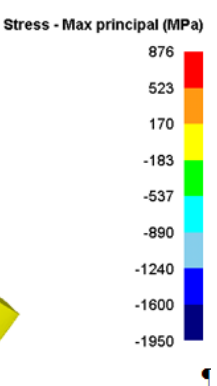

a - матрица с углом $\beta=0^{\circ} ;$ б - с углом $\beta=15^{\circ} ;$ в - с углом $\beta=25^{\circ} ;$ г - с углом $\beta=35^{\circ}$

На втором этапе деформирования (рис. 5) в матрице с углом $\beta=0^{\circ}$ значение главного напряжения $\sigma_{1}$ находится в диапазоне $190 \div 230$ МПа. С повышением значения угла $\beta$ значение главного напряжения $\sigma_{1}$ увеличивается. В матрице с углом $\beta=15^{\circ}$ значение $\sigma_{1}$ равно $250 \div 300$ МПа; в матрице с углом $\beta=25^{\circ}$ оно равно $290 \div 330 \mathrm{MПа;} \mathrm{в} \mathrm{матрице} \mathrm{с} \mathrm{углом} \beta=35^{\circ}$ оно равно $330 \div 390$ МПа.

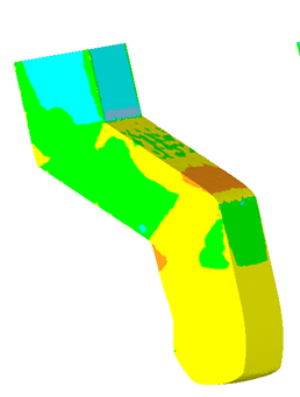

a

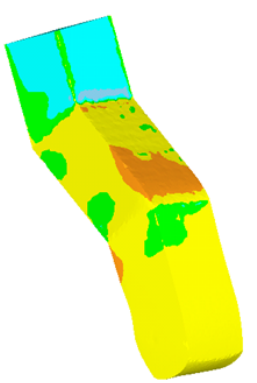

6

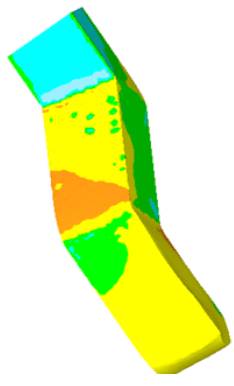

B
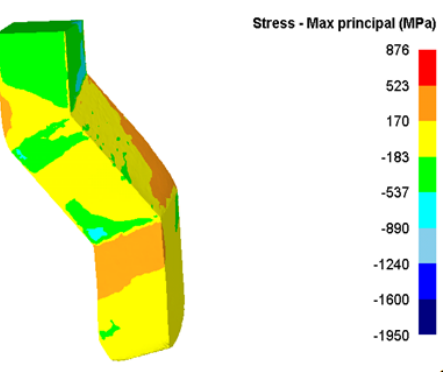

$\Gamma$

a - матрица с углом $\beta=0^{\circ} ;$ б - с углом $\beta=15^{\circ} ;$ в - с углом $\beta=25^{\circ} ;$ г - с углом $\beta=35^{\circ}$

Рис. 5. Распределение главного напряжения $\sigma_{1}$ на втором этапе деформирования 


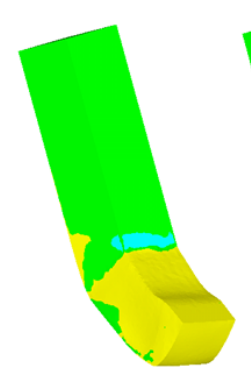

a

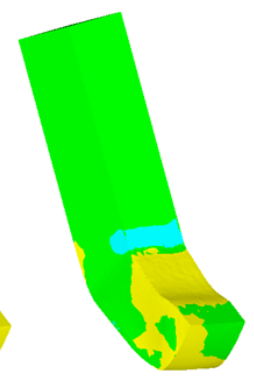

6

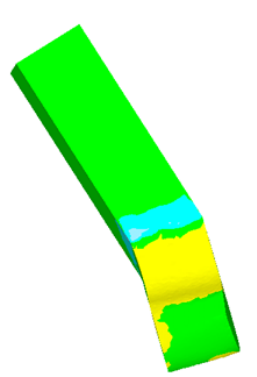

B

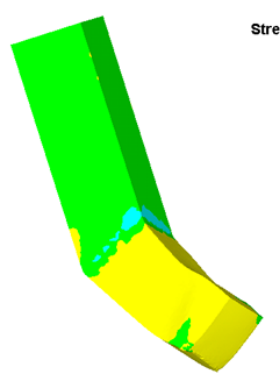

$\Gamma$

a- матрица с углом $\beta=0^{\circ} ; б-$ с углом $\beta=15^{\circ} ;$ в - с углом $\beta=25^{\circ} ;$ г - с углом $\beta=35^{\circ}$

Рис. 6. Распределение главного напряжения $\sigma_{3}$ на первом этапе деформирования

Главное напряжение $\sigma_{3}$ является преимущественно сжимающим, поэтому при его рассмотрении на заготовке отчетливо видны зоны напряжений с отрицательными значениями.

На первом этапе деформирования (рис. 6) в матрице с углом $\beta=0^{\circ}$ значение главного напряжения $\sigma_{3}$ находится в диапазоне $-350 \div-380$ МПа. С повышением значения угла $\beta$ значение главного напряжения $\sigma_{3}$ увеличивается (в своем абсолютном значении). В матрице с углом $\beta=15^{\circ}$ значение $\sigma_{3}$ равно $-440 \div-480$ МПа; в матрице с углом $\beta=25^{\circ}$ оно равно $-480 \div-520$ МПа; в матрице с углом $\beta=35^{\circ}$ оно равно $-560 \div-590$ МПа.

На втором этапе деформирования (рис. 7) в матрице с углом $\beta=0^{\circ}$ значение главного напряжения $\sigma_{3}$ находится в диапазоне $-380 \div-420$ МПа. С повышением значения угла $\beta$ значение главного напряжения $\sigma_{3}$ увеличивается. В матрице с углом $\beta=15^{\circ}$ значение $\sigma_{3}$ равно $540 \div-580$ МПа; в матрице с углом $\beta=25^{\circ}$ оно равно $-580 \div-620$ МПа; в матрице с углом $\beta=35^{\circ}$ оно равно $-680 \div-720$ МПа.

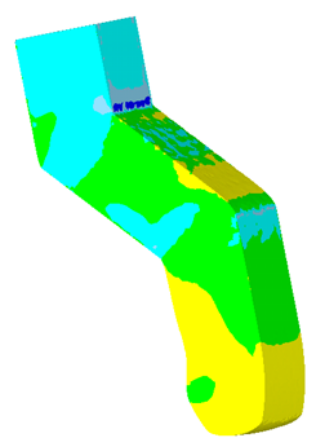

a

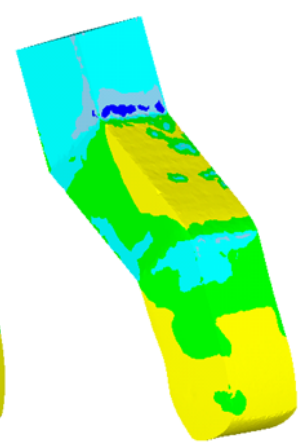

б

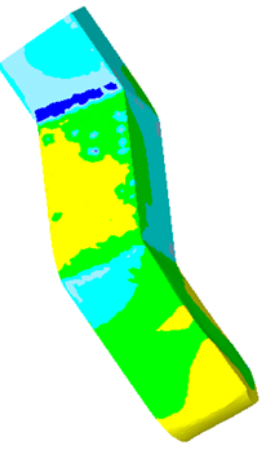

B

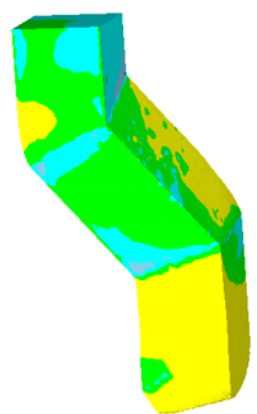

Stress - Min principal (MPa)

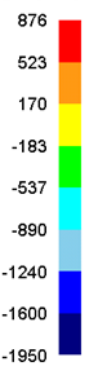

$\Gamma$

a - матрица с углом $\beta=0^{\circ} ;$ б - с углом $\beta=15^{\circ}$; в - с углом $\beta=25^{\circ} ;$ г - с углом $\beta=35^{\circ}$

Рис. 7. Распределение главного напряжения $\sigma_{3}$ на втором этапе деформирования

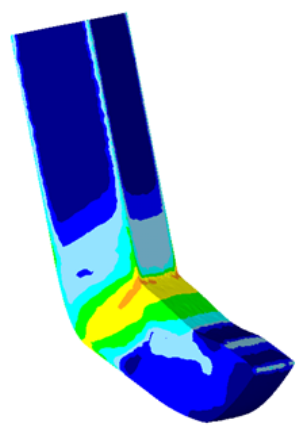

a

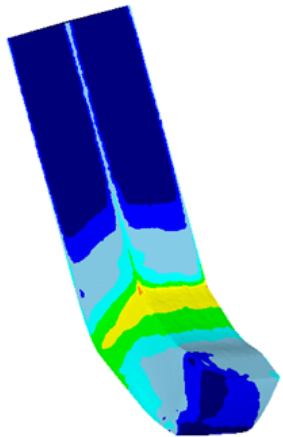

6

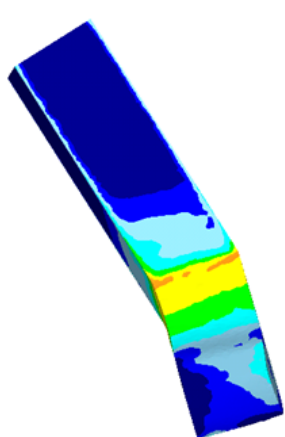

B

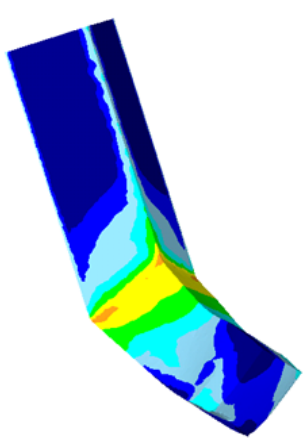

Stress - Effective (MPa)

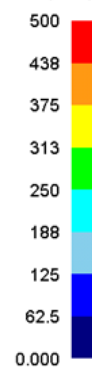

$\Gamma$

a - матрица с углом $\beta=0^{\circ} ; \sigma-$ с углом $\beta=15^{\circ} ;$ в - с углом $\beta=25^{\circ} ;$ г - с углом $\beta=35^{\circ}$

Рис. 8. Распределение эквивалентного напряжения на первом этапе деформирования 
Эквивалентное напряжение является условной величиной, которая включает в себя все компоненты тензора напряжений. Являясь подкоренным выражением, имеет только положительные значения. Смысл данного параметра состоит в том, чтобы показать в какойлибо точке деформируемого тела среднюю величину всех действующих напряжений (так называемая интенсивность напряжений).

На первом этапе (рис. 8) распределение эквивалентного напряжения находится преимущественно в зоне стыка первого и второго каналов. В матрице с углом $\beta=0^{0}$ в пристыковочной зоне значение эквивалентного напряжения колеблется в диапазоне $120 \div 180$ МПа. В зоне стыка каналов значение эквивалентного напряжения возрастает и находится в диапазоне $280 \div 350$ МПа. С повышением значения угла $\beta$ распределение эквивалентного напряжения по длине заготовки изменяется, протяженность очага деформации возрастает за счет дополнительного диагонального течения металла, а его численное значение увеличивается изза дополнительных сил подпора, действующих в третьей плоскости. В матрице с углом $\beta=15^{0}$ значение эквивалентного напряжения в пристыковочной зоне равно $130 \div 195$ МПа; в зоне стыка каналов - 300 $\div 370$ МПа. В матрице с углом $\beta=25^{0}$ значение эквивалентного

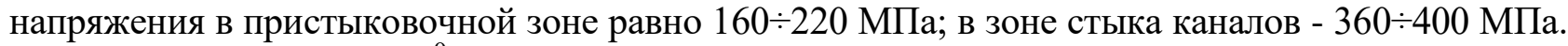
В матрице с углом $\beta=35^{0}$ значение эквивалентного напряжения в пристыковочной зоне рав-

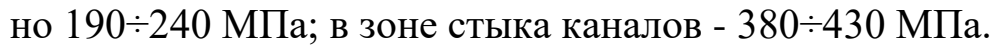

На втором этапе (рис. 9) распределение эквивалентного напряжения находится в двух ярко выраженных зонах: в зоне стыка первого и второго каналов и в зоне стыка второго и третьего каналов. В матрице с углом $\beta=0^{\circ}$ в пристыковочной зоне значение эквивалентного напряжения колеблется в диапазоне $180 \div 200$ МПа. В зоне стыка каналов значение эквивалентного напряжения возрастает и находится в диапазоне $420 \div 450$ МПа. С повышением значения угла $\beta$ численное значение эквивалентного напряжения увеличивается. В матрице с углом $\beta=15^{\circ}$ значение эквивалентного напряжения в пристыковочной зоне равно $220 \div 250$ МПа; в зоне стыка каналов - 430 $\div 470$ МПа. В матрице с углом $\beta=25^{\circ}$ значение эквивалентного напряжения в пристыковочной зоне равно $260 \div 320 \mathrm{MПа;} \mathrm{в} \mathrm{зоне} \mathrm{стыка} \mathrm{каналов}$ - 460 490 МПа. В матрице с углом $\beta=35^{\circ}$ значение эквивалентного напряжения в пристыко-

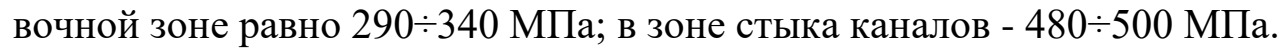

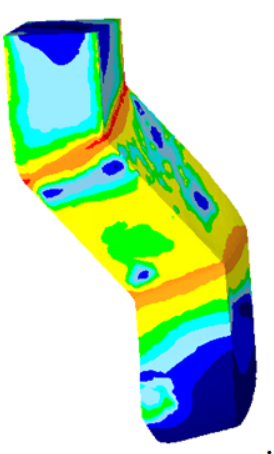

$\mathrm{a}$

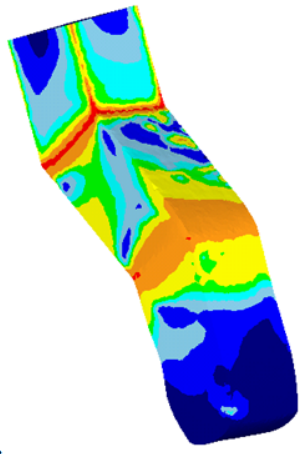

б

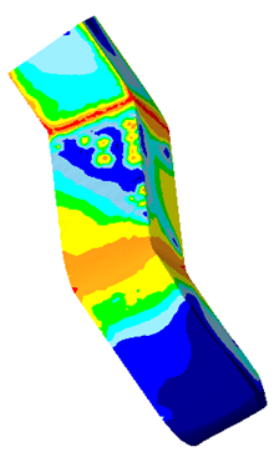

B

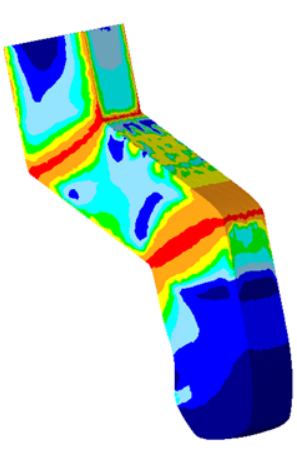

$\Gamma$

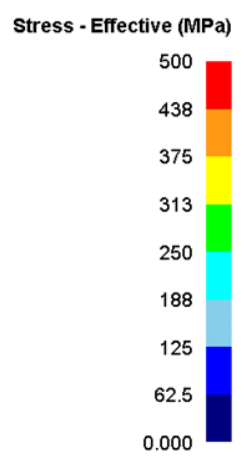

$\mathrm{a}$ - матрица с углом $\beta=0^{\circ} ; \sigma$ - с углом $\beta=15^{\circ} ;$ в - с углом $\beta=25^{\circ} ;$ г - с углом $\beta=35^{\circ}$

Рис. 9. Распределение эквивалентного напряжения на втором этапе деформирования

Как видно из данных, представленных на рис. 2-9, с увеличением величины угла $\beta$ все характеристики напряженно-деформированного состояния увеличиваются в своем абсолютном значении.

Из-за отсутствия обжатия в РКУ-матрицах как такового, рост эквивалентной деформации в данном процессе происходит исключительно за счет реализации схемы сдвига на наклонных плоскостях, соединяющих соседние каналы. Поэтому ключевым фактором, определяющим возникающий уровень деформаций за один проход, является величина угла 
(в нашем случае - углов) наклона данных плоскостей к входному и выходному вертикальным каналам. При увеличении угла $\beta$ с 0 до 35 градусов происходит рост эквивалентной деформации в среднем на $22 \div 25 \%$

Компоненты главных напряжений изменяют свои значения согласно своим характеристикам тензора напряжений - компоненты $\sigma_{1}$ и $\sigma_{3}$, являясь преимущественно однородными параметрами, имеют в основном положительные и отрицательные значения соответственно, причем величины $\sigma_{1}$ и $\sigma_{3}$ для первого и второго этапов близки по своему значению.

Эквивалентные напряжения имеют схожее распределение в обеих зонах - с увеличением угла $\beta$ происходит увеличение данного параметра. Однако изменение в зонах стыка каналов идет более плавно, чем в пристыковочных зонах. Это объясняется тем, что зоны стыка каналов, где собственно реализуются сдвиговые деформации, имеют довольно малую протяженность по сравнению с длинами каналов матрицы. В результате концентрация напряжения в этих зонах происходит в строго заданных промежутках, чего нельзя сказать о пристыковочных зонах, протяженность которых может доходить до половины длины каналов.

Исходя из этого, можно сделать вывод о том, что наиболее оптимальная схема деформации возникает при повышении угла $\beta$, т. е. в матрице с углом $\beta=35^{\circ}$.

Изучение сил деформирования

Для полноты исследования необходимо также оценить возникающую силу деформирования, поскольку чрезмерно большое значение этого параметра влечет за собой большие энергозатраты и может привести к поломке оборудования. Были построены графики возникающих сил, один из которых представлен на рис. 10, на них фиксировались значения силы для первого и второго этапов деформирования. Для удобства значения сил были представлены в виде сводной диаграммы (рис. 11).

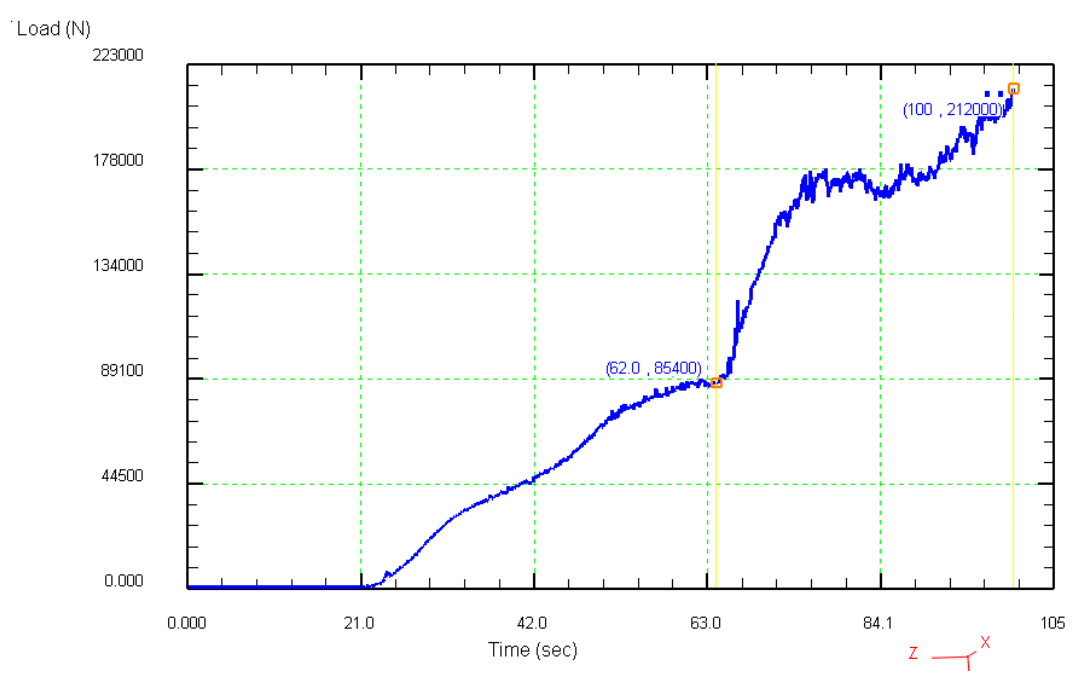

Рис. 10. Сила деформирования в матрице с углом $\beta=0^{\circ}$

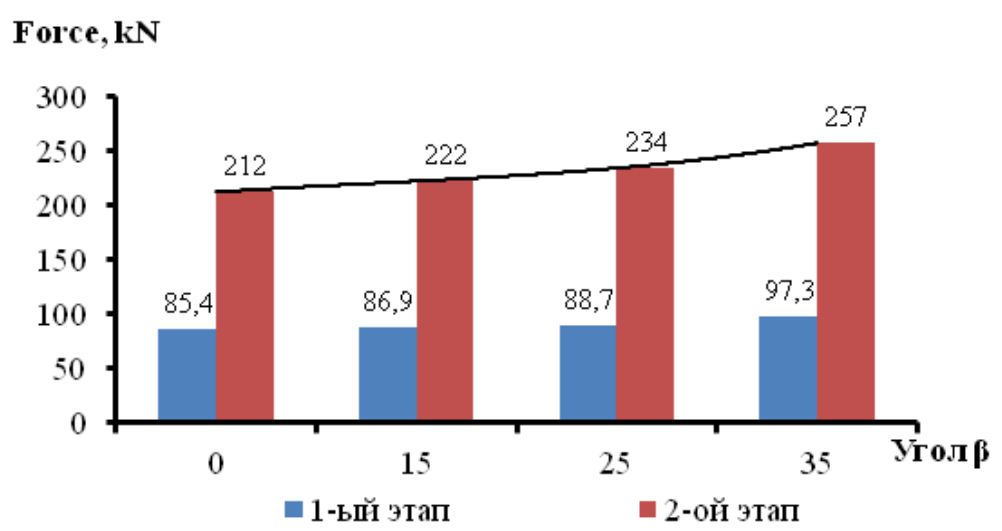

Рис. 11. Сводная диаграмма сил деформирования 
Как видно из диаграммы, при увеличении значения угла $\beta$ сила деформирования возрастает на обоих этапах. Увеличение силы происходит последовательно на 10, 12 и 23 кН, что говорит об экспоненциальном характере роста силы в зависимости от величины угла $\beta$. Максимальная величина силы 257 кН, возникающая при использовании матрицы с углом $\beta=35^{\circ}$ превышает стандартное значение для $\beta=0^{\circ}$ лишь на $22 \%$. Поэтому, оптимальным вариантом для осуществления равноканального ступенчатого прессования с течением металла в 3 плоскостях можно считать матрицу с углом $\alpha=135^{\circ}$ и углом $\beta=35^{\circ}$.

\section{ВЫВОДЫ}

В работе представлены результаты компьютерного моделирования процесса деформирования в равноканальной ступенчатой матрице, отличительной особенностью которой является расположение промежуточного канала под двумя углами к входному и выходному каналам. Рассмотрены модели со значениями угла наклона в продольном направлении (угол $\alpha) 135$ градусов и углами наклона в поперечном направлении (угол $\beta)$ 15, 25 и 35 градусов. Анализ параметров напряженно-деформированного состояния и возникающих сил деформирования показал, что наиболее оптимальная схема деформации возникает при повышении угла $\beta$, т. е. в матрице с углом $\beta=35^{\circ}$.

\section{СПИСОК ИСПОЛЬЗОВАННОЙ ЛИТЕРАТУРЫ}

1. Способ прессования: а. с. СССР № 575892. Сегал В. М. 1977.

2. Shaeri M. H., Salehi M. T., Seyyedein S. H., Abutalebi M. R., Park J. K. Microstructure and mechanical properties of Al-7075 alloy processed by equal channel angular pressing combined with aging treatment. Materials \& Design. 2014. 57, pp. 250-257.

3. Zhang Xiaohua, Liu Xiaojing, Wang Jingze, Cheng Yuansheng. Effect of route on tensile anisotropy in equal channel angular pressing. Materials Science and Engineering A. 2016. 676, pp. 65-72.

4. Wei W., Wang S.L., Wei K.X., Alexandrov I.V., Du Q.B., Hu J. Microstructure and tensile properties of $\mathrm{Cu}-\mathrm{Al}$ alloys processed by ECAP and rolling at cryogenic temperature. Journal of Alloys And Compounds. 2016. 678, pp. 506-510.

5. Mostaed E., Fabrizi A., Bonollo F., Vedani M. Microstructural, texture, plastic anisotropy and superplasticity development of ZK60 alloy during equal channel angular extrusion processing. Metallurgia Italiana. 2015. 11-12, pp. 5-12.

6. Zhao Xiaolian, Chen Na, Zhao Ningning. Numerical Simulation of equal channel angular pressing for multipass in different routes. Applied Mechanics and Materials. 2012. 268-270, pp. 373-377.

7. Gzyl M., Rosochowski A., Yakushina E., Wood P., Olejnik L. Route Effects in I-ECAP of AZ31B Magnesium Alloy. Key Engineering Materials. 2013. 554-557, pp. 876-884.

8. Lezhnev S. N., Toleuova As. R., Panin E. A. Modeling and study of the process of billets extrusion with additional back-pressure in equal channel step matrix. International Journal «Machines, Technologies, Materials». 11/2015, pp. 20-22.

9. Tavakkoli V., Afrasiab M., Faraji G., Mashhadi M. M. Severe mechanical anisotropy of high-strength ultrafine grained $\mathrm{Cu}-\mathrm{Zn}$ tubes processed by parallel tubular channel angular pressing (PTCAP). Materials Science and Engineering: A. 2015. 625, pp. 50-55.

10. Raab G. I., Fakhretdinova E. I., Valiev R. Z., Trifonenkov L. P., Frolov V. F. Computer study of the effect of tooling geometry on deformation parameters in the plastic shaping of aluminum wire rod by Multi-ECAP-Conform. Metallurgist. 2016. 59. 11-12, pp. 1007-1014.

11. Chembarisova R. G., Aleksandrov I. V. Simulation of the Elastoplastic Behavior of Grade-4 Ti in the ECAP-C Process. Metal Science and Heat Treatment. 2016. 58. 3-4, pp. 236-244.

12. Корчунов А. Г., Чукин М. В., Полякова М. А., Емалеева Д. Г. Принципы проектирования непрерывного способа получения стальной проволоки с ультрамелкозернистой структурой. Вестник Магнитогорского государственного технического университета им. Г. И. Носова. 2011. 1. С. 43-46.

13. Фастыковский А. Р. Экспериментальное изучение процесса прокатки - прессования. Кузнечноштамповочное производство. 2010. 11. С. 11-14.

14. Valiev R. Z., Langdon T. G. Principles of equal-channel angular pressing as a processing tool for grain refinement. Progress in Materials Science. 2006. 51, pp. 881-981.

15. Найзабеков А. Б., Ашкеев Ж. А., Лежнев С. Н., Толеуова А. Р. Исследование процесса деформирования заготовок в равноканальной ступенчатой матрице. Изв. вузов. Черная металлургия. 2005. 2. С. 16-18. 


\section{REFERENCES}

1. Pat. 575892. USSR. Pressing method. Segal V.M. 1977.

2. Shaeri M.H., Salehi M.T., Seyyedein S.H., Abutalebi M.R., Park J.K. Microstructure and mechanical properties of Al-7075 alloy processed by equal channel angular pressing combined with aging treatment. Materials \& Design. 2014. 57, pp. 250-257.

3. Zhang Xiaohua, Liu Xiaojing, Wang Jingze, Cheng Yuansheng. Effect of route on tensile anisotropy in equal channel angular pressing. Materials Science and Engineering: A. 2016. 676, pp. 65-72.

4. Wei W., Wang S.L., Wei K.X., Alexandrov I.V., Du Q.B., Hu J. Microstructure and tensile properties of CuAlloys processed by ECAP and rolling at cryogenic temperature. Journal of Alloys and Compounds. 2016. 678, pp. 506-510.

5. Mostaed E., Fabrizi A., Bonollo F., Vedani M. Microstructural, texture, plastic anisotropy and superplasticity development of ZK60 alloy during equal channel angular extrusion processing. Metallurgia Italiana. 2015. 11-12, pp. 5-12.

6. Zhao Xiaolian, Chen Na, Zhao Ningning. Numerical Simulation of equal channel angular pressing for multipass in different routes. Applied Mechanics and Materials. 2012. 268-270, pp. 373-377.

7. Gzyl M., Rosochowski A., Yakushina E., Wood P., Olejnik L. Route Effects in I-ECAP of AZ31B Magnesium Alloy. Key Engineering Materials. 2013. 554-557, pp. 876-884.

8. Lezhnev S.N., Toleuova As.R., Panin E.A. Modeling and study of the process of billets extrusion with additional back-pressure in equal channel step matrix. International Journal "Machines, Technologies, Materials". 2015. 11, pp. 20-22.

9. Tavakkoli V., Afrasiab M., Faraji G., Mashhadi M.M. Severe mechanical anisotropy of high-strength ultrafine grained $\mathrm{Cu}-\mathrm{Zn}$ tubes processed by parallel tubular channel angular pressing (PTCAP). Materials Science and Engineering: A. 2015. 625, pp. 50-55.

10. Raab G.I., Fakhretdinova E.I., Valiev R.Z., Trifonenkov L.P., Frolov V.F. Computer study of the effect of tooling geometry on deformation parameters in the plastic shaping of aluminum wire rod by Multi-ECAP-Conform. Metallurgist. 2016. 59. 11-12, pp. 1007-1014.

11. Chembarisova R.G., Aleksandrov I.V. Simulation of the Elastoplastic Behavior of Grade-4 Ti in the ECAP-C Process. Metal Science and Heat Treatment. 2016. 58. 3-4, pp. 236-244.

12. Korchunov A.G., Chukin M.V., Polyakova M.A., Emaleeva D.G. Principles of designing a continuous method for producing steel wire with an ultrafine-grained structure. Bulletin of the Magnitogorsk State Technical University G.I. Nosov. 2011. 1, pp. 43-46. (in Russian).

13. Fastykovsky A.R. Experimental study of the rolling - pressing process. Forging and Stamping Production. 2010. 11, pp. 11-14. (in Russian).

14. Valiev R.Z., Langdon T.G. Principles of equal-channel angular pressing as a processing tool for grain refinement. Progress in Materials Science. 2006. 51, pp. 881-981.

15. Naizabekov A.B., Ashkeev Zh.A., Lezhnev S.N., Toleuova A.R. Investigation of the deformation process of blanks in an equal-channel stepped matrix. Izvestiya. Ferrous Metallurgy. 2005. 2, pp. 16-18. (in Russian).

Найзабеков А. Б. - д-р техн. наук, проф. РИИ;

Лежнев С. Н. - канд. техн. наук, проф. РИИ;

Панин Е. А. $\quad-\mathrm{PhD}$, доцент КГИУ.

РИИ - Рудненский индустриальный институт, г. Рудный, Казахстан.

КГИУ - Карагандинский государственный индустриальный университет, г. Темиртау, Казахстан.

E-mail: $\underline{\text { Sergey_legnev@mail.ru }}$ 